\title{
Significado das ações educativas na consulta de enfermagem para clientes renais crônicos e familiares
}

\author{
Meaning of educational actions in nursing consultations for chronic renal clients and relatives \\ Significado de las acciones educativas en la consulta de enfermería para clientes renales crónicos y familiares
}

\author{
Harlon França de Menezes'; Ann Mary Machado Tinoco Feitosa Rosas"; Alessandra Conceição Leite Funchal Camacho'"; \\ Flávia Silva de Souza'v; Benedita Maria Rêgo Deusdará Rodrigues ${ }^{v}$; Richardson Augusto Rosendo da Silva ${ }^{\text {vl }}$
}

\begin{abstract}
Resumo
Objetivo: compreender o significado das ações educativas na consulta de enfermagem para clientes renais crônicos em tratamento conservador e seus familiares. Método: estudo orientado pela Fenomenologia Social. Entre novembro de 2015 e abril de 2016 , realizaram-se entrevistas com 12 clientes renais crônicos de um ambulatório de nefrologia de um hospital universitário e seus familiares, localizado no estado do Rio de Janeiro, Brasil, após aprovação dos Comitês de Ética em Pesquisa da instituição proponente e coparticipante. As entrevistas foram transcritas e submetidas à análise de acordo com a literatura relacionada. Resultados: emergiram duas categorias: Expectativas ao ir à consulta de enfermagem e Cuidando do outro para enfrentar as adversidades impostas pela doença. Conclusão: o estudo permitiu compreender que, no cotidiano desses indivíduos, eles adquirem habilidade e prática para lidar com suas limitações. As relações que possuem possibilitam subsídios para o reconhecimento dos valores não apenas da clínica como do valor enfocado no cuidado relacional.

Descritores: Insuficiência renal crônica; enfermagem no consultório; educação em saúde; família.
\end{abstract}

\section{ABSTRACT}

Objective: to understand the meaning of educational actions in nursing consultations for chronic renal clients in conservative treatment and their relatives. Method: study guided by Social Phenomenology. Between November 2015 and April 2016,12 chronic renal clients and their relatives were interviewed at a university hospital nephrology clinic in Rio de Janeiro State, Brazil, after approval by the research ethics committees of the proposing and co-participant institutions. The interviews were transcribed and submitted to analysis framed by the related literature. Results: two categories emerged: expectations when going to a nursing consultation and Caring for the other to face the adversities imposed by the disease. Conclusion: the study allowed us to understand that, in their daily lives, these individuals acquire the ability and practice to cope with their limitations. Their relationships afford contributions to recognition not only for the values of the clinic, but also for the value focused on by relational care.

Descriptors: Chronic renal failure; nursing in the office; health education; family.

\section{RESUMEN}

Objetivo: comprender el significado de las acciones educativas en la consulta de enfermería para clientes renales crónicos en tratamiento conservador y sus familiares. Método: estudio orientado por la Fenomenología Social. Entre noviembre de 2015 y abril de 2016, se realizaron entrevistas junto a 12 clientes renales crónicos, y a sus familiares, de un ambulatorio de nefrología de un hospital universitario, ubicado en el estado de Río de Janeiro, Brasil, tras la aprobación de los Comités de Ética en Investigación de la institución proponente y coparticipante. Las entrevistas fueron transcritas y sometidas al análisis de acuerdo con la literatura relacionada. Resultados: emergieron dos categorías: Expectativas al ir a la consulta de enfermería, y Cuidando al otro para enfrentar las adversidades impuestas por la enfermedad. Conclusión: el estudio permitió comprender que, en el cotidiano de esos individuos, estos adquieren habilidad y práctica para lidiar con sus limitaciones. Las relaciones que poseen posibilitan subsidios para el reconocimiento de los valores, no sólo de la clínica, sino también del valor enfocado en el cuidado relacional.

Descriptores: Insuficiencia renal crónica; enfermería en el consultorio; educación en salud; familia.

\section{INTRODUÇÃO}

O aumento da prevalência de doenças crônicas é visto como um dos eventos mais importantes que as comunidades e os profissionais de saúde têm enfrentado neste século. Hábitos e comportamentos de saúde podem afetar drasticamente a prevalência dessas doenças, já que estão associadas com disparidades sociodemográficas significativas, considerando que, em geral, minorias raciais/étnicas e os mais pobres, muitas vezes, estão propensos a ter essas doenças em idade precoce ${ }^{1,2}$.

\footnotetext{
'Enfermeiro. Universidade Federal Fluminense. Escola de Enfermagem Aurora de Afonso Costa. Programa Acadêmico em Ciências do Cuidado em Saúde. Niterói, Rio de Janeiro, Brasil. E-mail: harlonmenezes@hotmail.com

"Enfermeira. Professora Associada. Universidade Federal do Rio de Janeiro. Escola de Enfermagem Anna Nery. Departamento de Metodologia da Enfermagem. Rio de Janeiro, Brasil. E-mail: annmaryrosas@hotmail.com

"'Enfermeira. Professora Adjunta. Universidade Federal Fluminense. Escola de Enfermagem Aurora de Afonso Costa. Departamento de Fundamentos de Enfermagem e Administração. Niterói, Rio de Janeiro, Brasil. E-mail: cicacamacho@gmail.com

IVEnfermeira. Mestre em Enfermagem. Universidade Federal do Rio de Janeiro. Hospital Universitário Clementino Fraga Filho. Rio de Janeiro, Brasil. E-mail: sflaviashu@gmail.com vEnfermeira. Professora Titular. Universidade do Estado do Rio de Janeiro. Departamento de Enfermagem Materno Infantil. Rio de Janeiro, Brasil. E-mail: benedeusdara@gmail.com v'Enfermeiro. Professor Associado. Universidade Federal do Rio Grande do Norte. Departamento de Enfermagem. Natal, Rio Grande do Norte, Brasil. E-mail:
} rirosendo@hotmail.com 
A Doença Renal Crônica (DRC) é uma anomalia da estrutura renal ou da função, presente há mais de três meses, com sérias implicações para a saúde. É prolongada, geralmente coexistindo com outras, a exemplo da hipertensão e do diabetes. Consequentemente, é preciso fazer uso de muitos comprimidos, por exemplo, o que impõe elevada carga pessoal e monetária, extensivamente, às suas famílias ${ }^{3}$.

A DRC tem crescido vertiginosamente em países do hemisfério norte ${ }^{1,4}$. No Brasil, até o momento, não existem informações epidemiológicas definitivas. Contudo, estima-se que aproximadamente três milhões de brasileiros apresentem a doença em estágio avançado, e o relatório atual evidencia a tendência para o aumento do número de pessoas em diálise, das taxas de incidência e prevalência de tratamento, considerando, particularmente, os últimos quatro anos ${ }^{5,6}$.

Diante dessa realidade, a prevenção da progressão da DRC precisa ser focada nos fatores de risco, por meio de orientações específicas que atinjam a realidade do cotidiano, como também de quem convive e troca decisões a respeito do agravo e tratamento. Nesse sentido, a equipe de saúde tem papel fundamental e atuante ao direcionar e sensibilizar seus clientes para o alcance da qualidade de vida.

A enfermagem, por meio de sua principal atividade assistencial, a consulta de enfermagem, possui em sua atuação ações preventivas comportamentais que fazem parte do seu cotidiano e que podem assumir também a conotação de dever e de responsabilidade para com o outro. Essas ações definem-se como intervenções orientadas a evitar o surgimento de agravos específicos, estimulando a melhoria das suas condições clínicas, psicológicas e sociais ${ }^{7}$.

Estudos dão ênfase à atuação da enfermagem na DRC, tais como no controle da pressão arterial, nos fatores de risco aceitos para progressão e na necessidade dos programas preventivos considerando que muitos aspectos sociodemográficos estão associados a disparidades na saúde $\mathrm{e}^{8-10}$.

Desse modo, o suporte dado na consulta, ainda na fase de tratamento conservador, poderá servir de subsídio para que os profissionais da saúde possam aprimorar o seguimento ambulatorial desses indivíduos, com eficaz adesão ao tratamento, levando a resultados clínicos consideráveis e, consequentemente, à qualidade de vida. Nesse contexto, a progressão clínica da doença se estabiliza e o cliente torna-se apto a agir de modo receptivo.

Sendo assim, a questão que norteou este estudo foi: de que maneira as ações educativas na consulta denotam para o cliente renal crônico e seu familiar cuidador? Assim, o objetivo foi compreender o significado das ações educativas na consulta de enfermagem para clientes renais crônicos em tratamento conservador e seus familiares cuidadores.

\section{REFERENCIAL TEÓRICO}

Para a Fenomenologia Social, o importante é não separar os sujeitos do fenômeno, mas reuni-los indissociavelmente na estrutura intencional da experiência. Sendo assim, dá-se a fim de elucidar a estrutura significativa, ou seja, o conjunto dos significados subjetivos que constituem o mundo da vida, tendo como fundamento que toda ação que o sujeito desenvolve tem um sentido intencional e busca aí atender às suas expectativas ${ }^{11}$.

Nesse sentido, a aderência desse referencial ao estudo diz respeito ao cuidado à saúde que é originado a partir da interação entre as pessoas (intersubjetividade) e é sempre dotado de intenção (intencionalidade). Dessa forma, considera-se que as primeiras experiências de cuidado ocorrem no mundo da vida (mundo social) e são adquiridas com familiares e pessoas próximas. A estas experiências são acrescidas, ao longo da vida, as vivências pessoais e o conhecimento transmitido pelos profissionais acerca do cuidado à saúde (acervo de conhecimentos). O acervo de conhecimentos e a posição dos atores na esfera social (situação biográfica) permitem o estabelecimento de metas comuns para a ação social ${ }^{12}$.

Para o referencial, o cuidado é socialmente construído e tem origem no contexto existencial, sendo impulsionado pelas ações da motivação humana. Os motivos que se relacionam aos projetos do futuro são chamados de "motivos para" e aqueles que se fundamentam no acervo de conhecimentos e na experiência vivida no âmbito biopsicossocial do sujeito são denominados "motivos porque" 11 .

Neste estudo, é abordada a intencionalidade, ou seja, os projetos futuros de clientes e seus cuidadores por meio dos motivos-para.

\section{Metodologia}

A região de inquérito foi no mundo da vida de clientes renais crônicos e seus cuidadores que participam da consulta de enfermagem em um ambulatório de Nefrologia de um hospital universitário, localizado no município do Rio de Janeiro, Brasil.

Participaram deste estudo 12 clientes renais crônicos, maiores de 18 anos, atendidos no programa de tratamento conservador em estágios de III (pré-diálise) a $\mathrm{V}$ (não dialítico), e seus 12 respectivos familiares cuidadores, maiores de 18 anos, configurando, dessa maneira, os critérios de inclusão ${ }^{5}$. 0 motivo de inserir clientes em estágios já avançados de DRC se dá em razão de que a vivência destes é permeada por um perfil clínico que influi nas necessidades de saúde. Foram excluídos os clientes que compareceram sozinhos à consulta, bem como aqueles que participaram da consulta pela primeira vez por não terem o 'vivido' da consulta.

Foram realizadas, entre novembro de 2015 a abril de 2016, entrevistas fenomenológicas, gravadas 
pelo pesquisador principal por meio de dispositivo de mídia, que duraram em média 25 minutos, nas salas de atendimento, sendo apresentadas as seguintes questões aos clientes: o que você espera quando vem à consulta de enfermagem no ambulatório de Nefrologia? E para os cuidadores: o que você espera da consulta de enfermagem no ambulatório de Nefrologia? O que você tem em vista quando vem com seu familiar com DRC e recebe orientações educativas durante a consulta de enfermagem para cuidá-lo durante o tratamento?

Os participantes foram contatados previamente a fim de que fossem agendadas data e hora para a realização das entrevistas e, assim, assinaram o Termo de Consentimento Livre Esclarecido, garantindo o anonimato. Os participantes estão identificados com a palavra Cliente e Cuidador, seguida pelo número de sequência da entrevista. $O$ encerramento da realização de novas entrevistas aconteceu no momento em que se observou a repetição do conteúdo das respostas de ambos os grupos participantes quanto as suas motivações (motivo-para).

Os depoimentos foram analisados segundo os passos de pesquisadores da fenomenologia social: leitura e releitura de cada depoimento, com o objetivo de identificar os aspectos relevantes referentes ao contexto do vivido dos clientes e dos cuidadores; identificação e posterior agrupamento dos aspectos significativos dos depoimentos em unidades de significado; síntese das unidades de significado para posteriormente compor as categorias. A discussão dos dados teve como eixo norteador a fenomenologia social e literaturas relacionadas à temática ${ }^{13,14}$.

Esta pesquisa foi aprovada pelos Comitês de Ética em Pesquisa da instituição proponente e coparticipante por meio dos pareceres de número 1.045.709 e 1.067.955, respectivamente.

\section{RESULTADOS}

Dentre os clientes entrevistados, sete eram homens, na faixa etária entre 21 e 81 anos, sendo seis casados, todos com hipertensão arterial sistêmica e sete com diabetes, sendo 08 clientes em estágio IV de DRC. Quanto à média da faixa etária dos cuidadores, estava entre 31 e 72 anos, sendo dez do sexo feminino, e os principais vínculos eram pela relação conjugal e parental.

As expectativas, anseios e os objetivos por meio da relação face a face, apreendida pelas ações educativas na consulta de enfermagem, foram descritas na atitude natural própria, seja dos clientes, seja dos seus familiares, tendo, assim, os depoimentos que convergiram em categorias concretas do vivido descritas a seguir.

\section{Expectativas ao ir à consulta de enfermagem}

Os clientes demonstraram que a consulta se dá como recurso de aprendizagem para a melhoria da qualidade de vida por meio das ações educativas que aprendem. Nesse sentido, entendem que a consulta com o enfermeiro proporciona orientação para enfren- tar e vivenciar a DRC, já que compreendem a proposta do tratamento conservador, a partir do esclarecimento de dúvidas, e, assim, clareiam as supostas crenças, sem deixar de lado as relações sociais recíprocas que afetam o mundo. Os depoimentos a seguir retratam essa expectativa.

Olha, eu espero que seja legal para as pessoas que precisam, não é? Foi engraçado porque eu venho aqui há tanto tempo e consegui entender mais minhas coisas. (Cliente 01)

[...] A enfermeira que me atendeu na primeira vez, foi maravilhosa. Explicou-me que podia tirar as dúvidas com ela. (Cliente 02)

[...] Espero que vocês consigam dar ajuda a todo mundo que precisa. Meu filho, essa doença me pegou de uma maneira surpresa e quando vi já estava passando mal e sentindo umas coisas estranhas. [...] (Cliente 04)

Ah, espero que dê para eu entender as coisas melhor, eu sei que não me cuido como deveria, mas as coisas andam piorando, urina com muita espuma, passando mal direto, todo inchado. Então é bom vocês orientarem. (Cliente 07)

Ajuda a ter saúde, não é? E o que esperava é isso mesmo, ser orientado, ser ajudado e ficar bem. (Cliente 10)

Eu tenho vontade de ter mais uma ajuda nas minhas coisas, sabe? (Cliente 12)

\section{Cuidando do outro para enfrentar as adversidades impostas pela doença}

Os cuidadores dos clientes compreendem que a consulta se manifesta como estratégia altruísta para a população. Desse modo, o sistema de relevâncias, ou seja, o conjunto das prioridades e preferências dos sujeitos favorece o controle da DRC, visto que eles classificam receber orientações "diferentes" e pertinentes, demonstrando que a vinda até a consulta é satisfatória e possibilita amparo com vistas a viverem bem para enfrentarem as adversidades com o seu ente. Os depoimentos retratam essa percepção.

Eu espero que todos vocês possam ajudar a gente a entender mais a doença. [...] (Cuidadora 01)

Eu quero que ela fique bem. Mesmo diante de tudo que ela já passou, eu sempre estive com ela para ajudá-la a passar por tudo isso e passar de maneira tranquila e que ela não sofra, então eu quero que ela se estabilize. (Cuidadora 03)

[...] Então quando temos alguma orientação diferente, sempre é importante para ela entender mais, e eu estar junto. Eu acho que a consulta de vocês deveria ter em todos os lugares. (Cuidadora 06)

Eu espero que vocês possam ajudar os outros no que mais aflige as pessoas. Eu estou em um momento meu filho, que tenho que tomar conta de tudo para não piorar as coisas. (Cuidadora 05)

Quando eu venho, eu quero saber das coisas e procurar saber como ajudar. A gente tem esclarecimento de como cuidar e isso é legal. (Cuidador 08) 
Eu tenho a vontade de fazer com que ele fique tranquilo e sem medo. E o que vocês orientam, ajudam muito a gente em casa. Muito! [...] (Cuidadora 12)

\section{DISCUSSÃo}

A partir dos depoimentos, foi possível compreender que os clientes e seus cuidadores possuem percepções a respeito da participação na consulta de enfermagem e da contribuição que dela advém durante o tratamento conservador. As ações educativas na consulta são almejadas para buscar compreensão acerca da doença e como estratégia de compartilhamento, em vista das necessidades de interação e apoio durante o tratamento.

Estudo aponta a consulta como instrumento capaz de abarcar não apenas necessidades específicas, mas configura como um espaço para o enfrentamento de outras demandas do cotidiano, como é o caso de estigmas e entraves que permeiam algumas doenças, desvelando aspectos socioculturais que também precisam ser abordados ${ }^{15}$.

Deste modo, o enfermeiro deve ser capaz de desenvolver sua criatividade e sensibilidade de forma ativa, sistemática e contínua para que possa identificar a quantidade e a qualidade do seu cuidado. Assim, permite que suas ações, tidas como um trabalho vivo em ato, sistematizadas e pautadas no conhecimento, bem como em experiências prévias, conceitos próprios e intuitivos, formas específicas de ver e descrever a prática, voltem-se para o cuidado dos indivíduos, proporcionando-lhes segurança, conforto e bem-estar necessários para auxiliar o ser humano a vivenciar o seu processo de saúde-doença ${ }^{16}$.

Nesse sentido, na DRC, os clientes atendidos no tratamento conservador por uma equipe preparada e estimulada precisam de ações resolutivas que visem as suas peculiaridades, bem como as de seus cuidadores, que abarquem ações educativas plausíveis para esse grupo e que incentivem para o apoio e o autocuidado. Desse modo, as ações educativas devem ser consideradas prioritárias nas políticas de prevenção, diagnóstico e tratamento precoce, permitindo desenvolver nas pessoas uma consciência crítica da causa de seus problemas de saúde ${ }^{16}$.

Fica evidente nos depoimentos que as ações educativas que aprendem nas consultas mostram-se necessárias e condizentes com a realidade de cada um deles, enfatizando que medidas de proteção e identificação de possíveis agravos são abordadas, sendo-lhes ensinado como superá-los no cotidiano que vivenciam.

A abordagem educativa tem como propósito esclarecer detalhes relevantes acerca da doença e ensinar a adoção das práticas de autogestão dela, de forma acessível e dialógica, com a participação do doente, já que ele precisa conhecer e entender as formas de cuidar de si sem a imposição de métodos ou dificuldades, sendo um ser ativo no próprio tratamento. É relevante atribuir ao doente o perfil de agente ativo e participante do programa educativo, auxiliando-o na recuperação e/ ou adaptação imposta pela DRC ${ }^{17}$.

Estudos internacionais revelam que é promissor o suporte adjuvante dos enfermeiros em nefrologia, já que tem sido demonstrada a redução da taxa de progressão da DRC e na melhora das funções renais. Os resultados ainda mostraram melhorias mensuráveis em comportamentos de saúde, além de uma redução da duração e número de internações hospitalares ${ }^{9,18}$.

Os estudos ainda complementam que é relevante o apoio da viabilidade e a eficácia potencial de um modelo integrado de cuidados para a gestão da DRC, isto porque durante o curso de intervenções lideradas por enfermeiros houve melhora no controle dos fatores de risco aceitos para progressão da DRC. A adesão do cliente à medicação e o aconselhamento para o estilo de vida melhoram diante da intervenção integrada, indicando que clientes considerados de alto risco estão, por vezes, dispostos a envolver-se em modificações de estilo de vida quando recebem apoio e educação adequados sobre a gestão da sua condição combinada com a capacitação através da melhoria das competências de autocuidado ${ }^{9,18}$.

Assim, a equipe só possibilitará mudanças de comportamento se entender que a singularidade do ser humano só pode ser compreendida quando se tem contato direto com este. Os profissionais, ao se interessarem pela biografia de cada cliente, passam a conhecer o seu universo, independente da sua configuração, e ajudam a construir o modelo de cuidado. Isto quer dizer que interesses privados podem estar no mesmo contexto dos interesses de um grupo ${ }^{12}$.

Dentro do contexto biográfico, torna-se relevante a capacidade de o indivíduo obter, processar e compreender informações e serviços básicos de saúde necessários para tomar decisões pertinentes à própria saúde e aos cuidados médicos. É preciso considerar que o estresse decorrente do diagnóstico de uma doença grave e a sobrecarga de informações a serem seguidas contribuem para o aprendizado inadequado e, consequentemente, desencadeiam desfechos clínicos negativos. Todavia, o entendimento do cliente sobre as informações relativas à sua doença deve ser avaliado a cada consulta na tentativa de eliminar as disparidades de saúde ${ }^{19}$.

Pode-se compreender, então, que a as ações educativas precisam atender não só à capacidade de compreensão dos clientes renais crônicos, mas até a de seus cuidadores, haja vista a necessidade de colocá-los como atores de suas histórias. Assim, a experiência no ambulatório confluiu na utilidade de encaminhar os clientes e seus cuidadores ao Serviço da Nefrologia para que pudessem conhecer os passos de uma terapia renal substitutiva, por meio da hemodiálise e da diálise peritoneal, e assim desvelar crenças que permeavam o cotidiano desses indivíduos.

Desse modo, estudo realizado no sul do Brasil revelou que quanto mais cedo os clientes em estágios 
avançados forem preparados e conduzidos, pode-se evitar procedimentos de urgência e, para tanto, precisam fazer a melhor escolha quanto ao tratamento. Clientes renais crônicos em tratamento conservador demonstram conhecer os riscos de complicações perante as orientações recebidas e o esclarecimento da sua condição de saúde e comprometimento com as exigências para a manutenção do tratamento conservador ${ }^{20}$.

Assim, quando o cliente decide como quer e por onde seguir com seu tratamento, muitos deles têm o apoio de seus cuidadores próximos, sejam estes indivíduos vinculados como membros da própria família, como aqueles adquiridos pela vida social. A propósito, alguns participantes deste estudo contavam com a colaboração de pessoas que mesmo não tendo um vínculo parental agiam de modo atuante no mundo da vida do doente renal, comportando-se em todas as decisões.

Estudo realizado no interior de São Paulo que objetivou avaliar o suporte social e emocional de pessoas em tratamento hemodialítico revelou que entre as fontes mais frequentes de suporte encontrava-se o apoio proveniente da família, parceiros, cônjuge, companheiro ou namorado e amigos ${ }^{21}$.

Os resultados deste estudo vão ao encontro da pesquisa realizada em São Paulo com hipertensos com e sem doença renal, mostrando que foi significante a associação de doença renal com estado civil, com predominância de indivíduos com companheiro nesse grupo. Os autores ainda referiram que a convivência em casal tem sido utilizada como indicativo de apoio familiar, o qual estaria relacionado com melhor adesão ao tratamento de doenças crônicas e melhores resultados em saúde ${ }^{22}$.

Assim, a presença do cuidador deve ser vista como essencial no processo de enfrentamento e continuação do tratamento. A presença do cuidador na consulta é essencial, pois ele pode aprender, tirar dúvidas, mostrar suas condições e vontades, ou seja, ser ouvido. Sendo assim, ao interagir com o cuidador, o enfermeiro consegue desvelar sentimentos e propor ações plausíveis de saúde por meio de um cuidado intersubjetivo.

Esse cuidado está ligado sociologicamente quando indica que a intersubjetividade é fundamental para se compreender a existência humana, buscando os significados subjetivos dos atos e fenômenos do desenvolvimento histórico construído pelo homem. Inserir-se no mundo significa também comunicar-se com ele e como comunicação pressupõe intersubjetividade, são essas as tipificações que sustentam toda a relação social na qual o indivíduo nasce ${ }^{12}$.

Nesse contexto, já é sabido que a vivência com doença crônica é permeada por modos de enfrentamento e encorajamento quando se descobrem doentes e necessitam de subsídios. A relação destes com seus cuidadores merece e ganha destaque nas pesquisas, contudo, na realidade vivida, muitos podem passar pela aflição de como seus entes lidam com a doença, assim como aqueles que passam por esses momentos com entusiasmo.
Estudo com clientes em tratamento dialítico revelou que os constructos de esperança, espiritualidade e otimismo precisam ser considerados na assistência prestada pelos profissionais de saúde para auxiliar no enfrentamento da doença e do tratamento de clientes renais crônicos, e que esses profissionais devem ter o compromisso de considerar o nível desses constructos nas avaliações em saúde ${ }^{23}$.

O enfrentamento direcionado na emoção ou no problema de clientes em tratamento dialítico caracterizou-se pelas estratégias de apego à religião/crença, busca de apoio da família, negação e esquiva. A resiliência surgiu como modo de aceitação e um novo significado às suas vivências, buscando a adesão ao tratamento e cuidado em uma tentativa de minimizar os problemas causados pela doença ${ }^{24}$.

A reação em face da doença e as estratégias utilizadas pelos indivíduos a partir daí parecem se constituir em importantes fatores que precisam ser considerados para a promoção da saúde e da qualidade de vida dessa clientela. A compreensão sobre as medidas de enfrentamento permitirá à equipe de saúde oferecer o apoio adequado, no intuito de prevenir atitudes de pessimismo e desânimo perante a convivência com a enfermidade ${ }^{24}$.

Evidencia-se que as informações e orientações acerca do processo de adoecimento precisam ser prestadas pessoal e individualmente para que os vínculos possam ser fortalecidos. Assim, é preciso preocupar-se com a questão do cuidado personalizado e acolhedor como promotor da autonomia da pessoa diante da importância deste para a melhoria das condições de vida, na medida em que a doença avança ${ }^{7,25,26}$.

Sendo assim, pode-se compreender que a consulta de enfermagem deve ser intensificada e fortalecida pelos serviços de saúde, de maneira que seja uma possibilidade de estratégia efetiva de adesão ao tratamento e que os profissionais envolvidos se comprometam com a assistência prestada, considerando que as dimensões do retorno de suas ações voltam-se para o crescimento das pessoas.

\section{CONCLUSÃo}

A realização do estudo ensejou o cotidiano dos clientes renais crônicos e de seus familiares, bem como a modificação positiva quando aprendem e adquirem, por meio de ações educativas, habilidades para lidar com suas limitações. Ademais, as relações que estes possuem permitem que reconheçam os valores de sua clínica, como prioritariamente o valor enfocado no cuidado relacional, seja com seu familiar, seja com o enfermeiro.

Este estudo apresentou limitações caracterizadas pela especificidade da população estudada e por ser em uma realidade local. Como contribuições, aponta a necessidade de se olhar para a atenção oferecida às pessoas com DRC, sendo que a pesquisa possibilita reflexões 
que poderão impulsionar mudanças na prevenção e no manejo das complicações crônicas da doença e subsidiar novas avaliações na área.

Assim, possibilita-se a construção de novas práticas em saúde, na perspectiva da percepção de outras formas de fazer e cuidar, diante do significado de sofrimentos e enfrentamentos cotidianos, em que a atenção clínica reconheça esses limites para romper com o modo instituído de intervir, potencializando, dessa forma, ações de promoção de saúde.

\section{REFERÊNCIAS}

1. Ghadam MS, Poorgholami F, Jahromi ZB, Parandavar N, Kalani N, Rahmanian E. Effect of self-care education by face-to-face method on the quality of life in hemodialysis patients (Relying on Ferrans and Powers Questionnaire). Glob J. Health. Sci. [Online]. 2016 [cited 30 Abr 2017]; 8 (6): 121-127. Available from: https://dx.doi.org/10.5539\%2Fgjhs.v8n6p121 2. Tuot DS, Velasquez A, McCulloch CE, Banerjee T, Zhu Y, Hsu CY, et al. The Kidney Awareness Registry and Education (KARE) study: protocol of a randomized controlled trial to enhance provider and patient engagement with chronic kidney disease. BMC nephrology. [Online]. 2015 [cited 2017 Abr. 30]; 16: 166. Available from: https://www.ncbi.nlm.nih.gov/pmc/ articles/PMC4618520/pdf/12882 2015_Article_168.pdf

3. Sontakke S, Budania R, Bajait C, Jaiswal K, Pimpalkhute S. Evaluation of adherence to therapy in patients of chronic kidney disease. Indian j. pharmacol. [Online]. 2015 [cited 2017 abr 30]; 47 (6): 66871. Available from: https://www.ncbi.nlm.nih.gov/pmc/articles/ PMC4689023/.

4. Waterman AD, Peipert JD, Goalby CJ, Dinkel KM, Xiao H, Lentine KL. Assessing transplant education practices in dialysis centers: comparing educator reported and medicare data. Clin. j. am. soc. nephrol. [Online]. 2015 [cited 2017 abr 30]; 10 (9):1617-25. Available from: https://www.ncbi.nlm.nih.gov/pmc/ articles/PMC4559519/

5. Paula EA, Costa MB, Colugnati FAB, Bastos RMR, Vanelli CP, Leite CCA, et al. Strengths of primary healthcare regarding care provided for chronic kidney disease. Rev. latinoam. enferm. [Online]. 2016 [cited 2017 Out 16]; 24: e2801. Available from: http://www.scielo.br/ pdf/rlae/v24/0104-1169-rlae-24-02801.pdf

6. Sesso RC, Lopes AA, Thomé FS, Lugon JR, Martins CT. Brazilian Chronic Dialysis Census 2014. J. bras. nefrol. [Online]. 2016 [cited 2017 fev 25]; 38 (1): 54-61. Available from: http://www.scielo.br/pdf/jbn/v38n1/01012800-jbn-38-01-0054.pdf

7. Roso CC, Beuter M, Jacobi CS, Pauletto MR, Timm AMB, Silva CT. Taking care of yourself: limits and possibilities in the conservative treatment of chronic renal failure. Rev. enferm. UFPE on line. [Online]. 2015 [cited 2017 fev 25]; 9 (2): 617-2. Available from: http://www.revista.ufpe. br/revistaenfermagem/index.php/revista/article/view/5826/pdf_7153 8. Cirera Segura F, Pérez BÁ, Sánchez FN, Martín EJL. El control de la presión arterial en la consulta de enfermedad renal crónica avanzada. Enferm nefrol. [Online]. 2014 [cited 2017 Out 16]; 17 (1): 35-38. Available from: http://scielo.isciii.es/pdf/enefro/v17n1/08_original5.pdf 9. Walker RC, Marshall MR, Polaschek NR. A prospective clinical trial of specialist renal nursing in the primary care setting to prevent progression of chronic kidney: a quality improvement report. BMC family pract. [Online]. 2014 [cited 2017 Fev 25]; 15:155. Available from: https://www. ncbi.nlm.nih.gov/pmc/articles/PMC4263018/

10. Green JA, Cavanaugh KL. Understanding the influence of educational attainment on kidney health and opportunities for improved care. Adv. chronic kidney dis. [Online]. 2015 [cited 2017 Fev 25]; 22 (1): 24-30. Available from: https://doi.org/10.1053/j.ackd.2014.07.004

11. Jesus MCP, Capalbo C, Merighi MAB, Oliveira DM, Tocantins FR, Rodrigues BM, et al. The social phenomenology of Alfred Schutz and its contribution for nursing. Rev. Esc. Enferm. USP. [Online]. 2013 [cited 2017 Fev 25]; 47 (3):736-41. Available from: http://www.scielo.br/pdf/ reeusp/v47n3/0080-6234-reeusp-47-3-00736.pdf

12. Silva MH, Jesus MCP, Oliveira DM, Merighi MAB. Unna's boot: expe- rience of care of people with venous ulcers. Rev. bras. enferm. [Online]. 2017 [cited 2017 Fev 25]; 70 (2): 349-56. Available from: http://www. scielo.br/pdf/reben/v70n2/pt 0034-7167-reben-70-02-0349.pdf 13. Jesus MCP, Silva MH, Cordeiro SM, Korchmar E, Zampier VSB, Merighi $M A B$. Understanding unsuccessful attempts to quit smoking: a social phenomenology approach. Rev. Esc. Enferm. USP. [Online]. 2016 [cited 2017 Fev 26]; 50 (1): 71-78. Available from: http://www.scielo.br/pdf/ reeusp/v50n1/pt_0080-6234-reeusp-50-01-0073.pdf

14. Costa PCP, Garcia APRF, Toledo VP. Welcoming and nursing care: a phenomenological study. Texto \& contexto enferm. [Online]. 2016 [cited 2017 Fev 26]; 25 (1): e4550015. Available from: http://www.scielo.br/ pdf/tce/v25n1/pt_0104-0707-tce-25-01-4550015.pdf

15. Oliveira DRC, Enders BC, Vieira CENK, Mariz LS. Assessment of nursing consultations for tuberculosis patients at primary health care. Rev. eletrônica enferm. [Online]. 2016 [cited 2017 Fev 26]; 18: e1153. Available from: http://dx.doi.org/10.5216/ree.v18.32593

16. Dantas CN, Santos VEP, Tourinho FSV. Nursing consultation as a technology for care in light of the thoughts of Bacon and Galimberti. Texto \& contexto enferm. [Online]. 2016 [cited 2017 Sep 30]; 25 (1): e2800014. Available from: http://www.scielo.br/pdf/tce/v25n1/ en_0104-0707-tce-25-01-2800014.pdf

17. Santos RLG, Oliveira DRF, Nunes MGS, Barbosa RMP, Gouveia VA. Evaluation of the knowledge of chronic renal patients in conservative treatment about dialytic modalities. Rev. enferm. UFPE on line. [Online]. 2015 [cited 2017 fev 26]; 9 (2): 651-60. Available from: http://www.revista.ufpe. br/revistaenfermagem/index.php/revista/article/view/7360/pdf_7169 18. Peeters MJ, van Zuilen AD, van den Brand JA, Bots ML, van Buren M, Ten Dam MA, Kaasjager KA, Ligtenberg G, Sijpkens YW, Sluiter HE, van de Ven PJ, Vervoort G, Vleming L, Blankestijn PJ, Wetzels JF. Nurse practitioner care improves renal outcome in patients with CKD. J. Am. Soc. Nephrol. [Online]. 2014 [cited 2017 fev 26]; 25 (2): 390-8. Available from: https://www.ncbi.nlm.nih.gov/pmc/articles/PMC3904557/

19. Sampaio HAC, Carioca AAF, Sabry MOD, Santos PM, Coelho MAM, Passamai MPB. Health literacy in type 2 diabetics: associated factors and glycemic control. Ciênc. saúde coletiva. [Online]. 2015 [cited 2017 Fev 26]; 20 (3): 865-874. Available from: http://www.scielo.br/pdf/csc/ v20n3/1413-8123-csc-20-03-00865.pdf

20. Roso CC, Beuter M, Bruinsma JL, Silva JH, Timm AMB, Pauletto MR. Clinical aspects of people with chronic renal failure in conservative treatment. Rev. RENE. [Online]. 2013 [cited 2017 Out 15]; 14 (6): 12011208. Available from: http://www.revistarene.ufc.br/revista/index.php/ revista/article/view/1380/pdf

21. Silva SM, Braido NF, Ottaviani AC, Gesualdo GD, Zazzetta MS, Orlandi FS. Social support of adults and elderly with chronic kidney disease on dialysis. Rev. latinoam. enferm. [Online]. 2016 [cited 2016 Out 15]; 24: e2752. Available from: http://www.scielo.br/pdf/rlae/v24/0104-1169rlae-24-02752.pdf

22. Pinho NA, Oliveira RCB, Pierin AMG. Hypertensive patients with and without kidney disease: assessment of risk factors. Rev. Esc. Enferm. USP. [Online]. 2015 [cited 2017 Out 15]; 49: 101-108. Available from: http://www.scielo.br/pdf/reeusp/v49nspe/en_1980-220X-reeusp49-spe-0101.pdf

23. Ottaviani AC, Souza ÉN, Drago NC, Mendiondo MSZ, Pavarini $\mathrm{SCl}$, Orlandi FS. Hope and spirituality among patients with chronic kidney disease undergoing hemodialysis: a correlational study. Rev. latinoam. enferm. [Online]. 2014 [cited 2017 Out 15]; 22 (2): 248-254. Available from: http://www.scielo.br/pdf/rlae/v22n2/pt_0104-1169rlae-22-02-00248.pdf

24. Silva RAR, Souza Neto VL, Oliveira GJN, Silva BCO, Rocha CCT, Holanda JRR. Coping strategies used by chronic renal failure patients on hemodialysis. Esc. Anna Nery Rev. Enferm. [Online]. 2016 [cited 2017 Out 15]; 20 (1): 147-154. Available from: http://www.scielo.br/pdf/ean/v20n1/ en_1414-8145-ean-20-01-0147.pdf

25. Araujo CRG, Rosas AMMTF, Menezes HF, Rodrigues BMRD. Teaching and learning in the gynecological brachytherapy nursing appointment: the nurses' perspectives. Rev. enferm. UERJ. [Online]. 2016 [cited 2018 Sep 26]; 24 (4): e18737. DOI: http://dx.doi.org/10.12957/reuerj.2016.18737 26. Ferraz RN, Maciel CG, Borba AKOT, Frazão IS, França VV. Health personnel's perceptions of factors influencing hemodialysis treatment adherence. Rev. enferm. UERJ. [Online]. 2017 [cited 2018 Sep 26]; 25:e15504. DOI: http://dx.doi.org/10.12957/reuerj.2017.15504 